\title{
Economic Cost of a Listeria monocytogenes Outbreak in Canada, 2008
}

\author{
M. Kate Thomas, Rachael Vriezen,, Jeffrey M. Farber, \\ Andrea Currie, Walter Schlech, ${ }^{3}$ and Aamir Fazil ${ }^{1}$
}

\begin{abstract}
Estimates of the economic costs associated with foodborne disease are important to inform public health decisionmaking. In 2008, 57 cases of listeriosis and 24 deaths in Canada were linked to contaminated delicatessen meat from one meat processing plant. Costs associated with the cases (including medical costs, nonmedical costs, and productivity losses) and those incurred by the implicated plant and federal agencies responding to the outbreak were estimated to be nearly $\$ 242$ million Canadian dollars (CAD, 2008). Case costs alone were estimated at approximately $\$ 2.8$ million (CAD, 2008) including loss of life. This demonstrates the considerable economic burden at both the individual and population levels associated with foodborne disease and foodborne outbreaks in particular. Foodborne outbreaks due to severe pathogens, such as Listeria monocytogenes and those that result in product recalls, are typically the most costly from the individual and/or societal perspective. Additional economic estimates of foodborne disease would contribute to our understanding of the burden of foodborne disease in Canada and would support the need for ongoing prevention and control activities.
\end{abstract}

\section{Introduction}

$\mathbf{I}$ N CANADA, IT IS ESTIMATED that there are 4 million episodes of domestically acquired foodborne illness each year, of which 178 are caused by Listeria monocytogenes (Thomas et al., 2013). The annual global burden of listeriosis was estimated to be more than 23,000 illnesses and almost 5500 deaths in 2010 (de Noordhout et al., 2014). Though it is a relatively rare disease, invasive listeriosis often results in severe symptoms including sepsis, encephalitis, and meningitis. Elderly or immunocompromised individuals, as well as pregnant women and their unborn children, are the most vulnerable to severe infection (Schlech and Acheson, 2000; WHO/FAO, 2004). The severity of symptoms and high casefatality rate make the study and control of L. monocytogenes important for food safety both in Canada and internationally.

In 2008, 57 cases of acquired listeriosis in Canada were linked to delicatessen meat contaminated with L. monocytogenes originating from an Ontario ready-to-eat meatprocessing plant (implicated meat-processing facility; IMP) (Currie et al., 2015). Forty-seven individuals were hospitalized due to listeriosis, and listeriosis was the underlying or contributing cause of 24 deaths. The median age of affected individuals was 78 years (range 29-98 years). Cases were reported in seven provinces, with the majority (72\%) occurring in Ontario. An outbreak of this magnitude places a significant burden on the individuals involved, the healthcare system, and on the implicated industry. In addition, government public health and food safety agencies at the local, provincial/territorial, and federal levels invest resources to investigate, respond to, and manage outbreaks as outlined in the Foodborne Illness Outbreak Response Protocol (Government of Canada, 2010).

Estimates of the costs associated with foodborne disease are important to inform public health decision-making (Rice, 2000). Such estimates have been generated internationally (Ryser and Marth, 1989; Buzby et al., 1996; Abelson et al., 2006; Kemmeren et al., 2006; Cressey and Lake, 2008; Haagsma et al., 2009; Scharff, 2010; Hoffmann et al., 2012; Mangen et al., 2015); however, few of these are Canadian studies (Todd, 1989a, b) or estimate the cost of a specific outbreak (Todd, 1985; Roberts et al., 1989, 2000). Examining the costs associated with outbreaks and exploring methods for this purpose will provide important information to support

\footnotetext{
${ }^{1}$ Public Health Agency of Canada, Guelph, Ontario, Canada.

${ }^{2}$ Health Canada, Ottawa, Ontario, Canada.

${ }^{3}$ Dalhousie University, Halifax, Nova Scotia, Canada.
}

(C) The Author(s) 2015; Published by Mary Ann Liebert, Inc. This Open Access article is distributed under the terms of the Creative Commons Attribution Noncommercial License (http://creativecommons.org/licenses/by-nc/4.0/) which permits any noncommercial use, distribution, and reproduction in any medium, provided the original author(s) and the source are credited. 
investment in prevention and control efforts related to outbreaks (Roberts, 2000). The goal of this article is to estimate the economic cost of the 2008 listeriosis outbreak including costs associated with the cases, federal outbreak response costs, and costs to the IMP. The outbreak response costs for the provincial/territorial and local level were not included due to a lack of available data but were likely to have been substantial.

\section{Methods}

To estimate the economic cost of the 2008 listeriosis outbreak, costs related to cases, federal outbreak response and the IMP were assessed. Detailed descriptions of the model inputs are provided in Supplementary Data S1-S4; Supplementary Data are available online at www.liebertpub.com/fpd

\section{Case costs}

Cost of illnesses. Case costs were classified into three categories: (1) Direct Healthcare Costs (DHC), or those medical costs that are a direct result of illness and requisite medical care, including diagnosis, hospitalization, general physician care, specialist care, and treatment; (2) Direct NonHealthcare Costs (DNHC), or those nonmedical costs borne directly by affected individuals, in particular transportation costs; and (3) Indirect Non-Healthcare Costs (INHC), or those nonmedical costs indirectly due to infections, in particular production losses to society resulting from illness (Supplementary Data S1). A modified friction cost method was used to calculate production losses resulting from shortterm absences, where the employee is likely to return to paid employment following illness. These were calculated as the estimated duration of the absence multiplied by the average daily wage in Canada in 2008. Production losses related to caregivers were not estimated due to data limitations.

Cost of deaths. The burden associated with deaths (part of INHC) was estimated in two ways. A value of statistical life (VSL) figure based on recommendations for Canadian policy analysis was used to estimate a monetary figure for the value to society of the loss of life resulting from this outbreak (Chestnut and De Civita, 2009). In addition, disability adjusted life years (DALYs), which provide an indicator of the loss of functional life due to morbidity and premature mortality, were calculated to provide an alternative, nonmonetary estimate of the burden associated with pain, suffering, and loss of life (Supplementary Data S4).

For each case, the following variables were extracted from the outbreak dataset (Currie et al., 2015) for this analysis: age, gender, province, symptom onset date (estimated by specimen collection date for four cases where onset date was not applicable or available), hospitalization as a result of listeriosis, hospital admission and discharge dates (where reported), clinical presentation and symptoms, institutional setting prior to illness (i.e., resident of a long-term care facility, hospital inpatient), whether or not the case died and if so, whether or not listeriosis caused or contributed to the death.

\section{Federal outbreak response costs}

The federal government outbreak response costs were estimated for the Public Health Agency of Canada, Health Canada and the Canadian Food Inspection Agency (Supplementary
Data S2). These costs included time (both regular and overtime hours) spent investigating, responding to and managing the outbreak, as well as costs associated with molecular subtyping of clinical isolates conducted at the National Microbiology Laboratory and food sample testing conducted by Health Canada. Outbreak response and laboratory costs for the provincial/territorial and local public health agencies were not considered due to a lack of available data.

Costs were broken down into two main time periods where possible for the departments reporting outbreak response costs. Time period 1 refers to the peak of the outbreak investigation and response activities (July 27-September 6, 2008) and time period 2 refers to the remainder of the outbreak investigation and response period (September 7October 25, 2008). Following time period 2, investigation and response activities returned to routine levels. When available, results are reported for each time period as well as both time periods combined.

\section{IMP costs}

Costs for the IMP associated with direct and indirect losses as well as legal fees were estimated based on publicly available information and personal communications (Supplementary Data S3). Minimal information was available regarding costs to industry associated with this outbreak, and thus there is a high level of uncertainty surrounding the estimated costs. Minimum and maximum costs were estimated based on all the available data. Additionally, information about the indirect costs of this outbreak to the ready-to-eat meats and related industries was not available and therefore is excluded from this estimate. The IMP costs described are likely an underestimate of the true economic impact of this outbreak to this specific establishment and the industry in general.

\section{Cost conversions}

All costs were obtained in Canadian dollars (CAD) and adjusted to 2008 prices using the Canadian Consumer Price Index (CPI) or where applicable using the appropriate corresponding component of the CPI (Supplementary Data S1).

\section{Uncertainty analysis}

To account for the uncertainty associated with the cost estimates, inputs were described using probability distributions. Empirical data were used wherever possible to inform these distributions, although expert opinion was solicited in instances where such information was unavailable.

For most inputs, PERT distributions were developed using the minimum, most likely, and maximum values of that input. For hospital costs and wages per day, available data were fitted to appropriate distributions (Supplementary Data S1-S3).

Final estimates were calculated using Monte Carlo simulation at 100,000 iterations, using the @ Risk software add-in for Microsoft Excel (Microsoft Corporation, Redmond, WA). The mean and $90 \%$ credible intervals were reported.

\section{Results}

Cost estimates were combined in the model to generate output distributions for the total cost and cost per case of the outbreak. Case costs, federal outbreak response costs, and the 
Table 1. Case Costs Associated with the 2008 Listeriosis Outbreak in Canada ${ }^{a}$

\begin{tabular}{|c|c|c|c|c|}
\hline & Mean total costs & $5 \%$ & $95 \%$ & Number of cases \\
\hline \multicolumn{5}{|l|}{ Direct healthcare costs } \\
\hline Hospital fees & $\$ 647,921$ & $\$ 377,262$ & $\$ 1,012,421$ & 57 \\
\hline General physician fees & $\$ 35,439$ & $\$ 30,615$ & $\$ 40,735$ & 57 \\
\hline Diagnosis/specialist/treatment costs & $\$ 67,891$ & $\$ 49,873$ & $\$ 87,904$ & 57 \\
\hline Total direct healthcare costs & $\$ 751,251$ & $\$ 478,272$ & $\$ 1,117,835$ & \\
\hline \multicolumn{5}{|l|}{ Indirect nonhealthcare costs } \\
\hline Lost productivity due to illness & $\$ 26,845$ & $\$ 9,869$ & $\$ 63,418$ & \\
\hline Total indirect nonhealthcare costs & $\$ 26,845$ & $\$ 9,869$ & $\$ 63,418$ & \\
\hline \multicolumn{5}{|l|}{ Direct nonhealthcare costs } \\
\hline Transportation costs to hospital & $\$ 797$ & - & - & 47 \\
\hline Transportation costs to general practitioner clinic & $\$ 41$ & $\$ 27$ & $\$ 53$ & 4 \\
\hline Total direct nonhealthcare costs & $\$ 838$ & $\$ 824$ & $\$ 850$ & \\
\hline Total cost of illness & $\$ 778,934$ & $\$ 503,867$ & $\$ 1,146,272$ & \\
\hline Cost of illness per case & $\$ 13,666$ & $\$ 8,840$ & $\$ 20,110$ & \\
\hline Total DALYs & 142 & 142 & 143 & \\
\hline DALYs per case & 2 & 2 & 3 & \\
\hline Total VSL values for deaths & $\$ 161,292,861$ & $\$ 115,026,682$ & $\$ 207,557,221$ & 24 \\
\hline Total case costs (including VSL for deaths) & $\$ 162,071,795$ & $\$ 115,825,039$ & $\$ 208,291,225$ & \\
\hline Cost per case (including VSL for deaths) & $\$ 2,843,365$ & $\$ 2,032,018$ & $\$ 3,654,232$ & \\
\hline
\end{tabular}

${ }^{a}$ Totals are based on stochastic distributions and thus column input values do not necessarily add exactly to totals.

DALYs, disability-adjusted life years; VSL, value of statistical life.

IMP costs are reported in Tables $1-3$ as the estimated mean and $90 \%$ credible interval values.

Case costs are reported both with and without the VSL associated with the premature deaths (Table 1). Total case costs, not including the VSL, are nearly $\$ 780,000$, of which hospital fees, not including the cost of diagnostics, specialist care, or medical treatment, account for $83 \%$ of the total. VSL values associated with the 24 deaths are $\$ 161,000,000$. If the burden related to deaths is estimated using DALYs, then pain and suffering can also be explicitly included. This total equals 142 DALYs. To account for an individual's preference for health benefits in the present rather than the future, it is common practice to discount the value of DALYs (Murray, 1994; Kemmeren et al., 2006). The number of discounted DALYs calculated in this study is 115 (Supplementary Data S4).
Federal-level outbreak response costs are reported in Table 2. Costs to Public Health Agency of Canada (PHAC) were estimated to be $\$ 862,000$ including approximately 19,500 regular and overtime hours of staff time. Costs for Health Canada were estimated at nearly $\$ 225,000$ and included 514 overtime hours. Costs for Canadian Food Inspection Agency (CFIA) were approximated based on values calculated for PHAC and were estimated to be nearly $\$ 1,300,000$.

Costs to the IMP are estimated to be approximately $\$ 77,250,000$ (range \$56,774,769-\$97,724,555).

The summary of costs is presented in Table 3. The overall total costs are approximately $\$ 242,000,000$ with the average cost per case estimated at $\$ 4.2$ million (CAD 2008) (including federal response costs, IMP costs, and case costs); case costs alone were estimated at approximately $\$ 2.8$ million (CAD 2008) (including deaths). Federal

Table 2. Federal Outbreak Response Costs Associated With THE 2008 Listeriosis OUtBreak IN CANADA ${ }^{a}$

\begin{tabular}{lccccc}
\hline & Mean total costs & $5 \%$ & $95 \%$ & Total hours & Total overtime hours \\
\hline PHAC & & & & & \\
$\quad$ Time 1: All except NML & $\$ 430,522$ & $\$ 418,680$ & $\$ 442,432$ & 9650 & 205 \\
Time 2: All except NML & $\$ 222,139$ & $\$ 211,523$ & $\$ 232,864$ & 5033 & 98 \\
Combined: All except NML & $\$ 652,661$ & $\$ 637,003$ & $\$ 668,452$ & 14,683 & 303 \\
NML: staff & $\$ 147,110$ & - & - & 4251 & 285 \\
NML: laboratory & $\$ 25,774$ & - & - & - & - \\
$\quad$ Combined: All PHAC & $\$ 862,703$ & $\$ 847,045$ & $\$ 878,494$ & 18,934 & 588 \\
Health Canada & $\$ 224,175$ & $\$ 210,242$ & $\$ 238,107$ & - & 514 \\
CFIA & $\$ 1,294,038$ & $\$ 1,025,776$ & $\$ 1,563,369$ & - & - \\
Total federal outbreak response costs & $\$ 2,380,928$ & $\$ 2,110,829$ & $\$ 2,652,494$ & 18,934 & 1102 \\
\hline
\end{tabular}

${ }^{a}$ Totals are based on stochastic distributions and thus column input values do not necessarily add exactly to totals.

PHAC, Public Health Agency of Canada; NML, National Microbiology Laboratory; CFIA, Canadian Food Inspection Agency. 
Table 3. Summary of Costs Associated with the 2008 Listeriosis Outbreak in Canada ${ }^{a}$

\begin{tabular}{lrrr}
\hline & Mean total costs & \multicolumn{1}{c}{$5 \%$} & \multicolumn{1}{c}{$95 \%$} \\
\hline Total case costs (including deaths) & $\$ 162,071,795$ & $\$ 115,825,039$ & $\$ 208,291,225$ \\
Total federal outbreak response costs & $\$ 2,380,928$ & $\$ 2,110,829$ & $\$ 2,652,494$ \\
Total costs to the implicated meat-processing facility & $\$ 77,249,999$ & $\$ 56,774,702$ & $\$ 97,724,604$ \\
Overall total costs & $\$ 241,702,723$ & $\$ 174,710,570$ & $\$ 308,668,323$ \\
Overall cost per case & $\$ 4,240,399$ & $\$ 3,065,098$ & $\$ 5,415,234$ \\
\hline
\end{tabular}

${ }^{a}$ Totals are based on stochastic distributions and thus column input values do not necessarily add exactly to totals.

outbreak response costs represent the smallest proportion (1\%) of the total cost, whereas case costs (including deaths) account for $67 \%$ of the total cost associated with the outbreak.

\section{Discussion}

The costs associated with the 2008 listeriosis outbreak in Canada were estimated to be nearly $\$ 242$ million. This is likely a conservative estimate for several reasons. First, costs incurred by local and provincial/territorial public health and food safety authorities were not included. Additionally, costs for the CFIA were estimated based on the estimated cost to PHAC, which represents a minimum cost, as CFIA's mandate during a foodborne outbreak is different from PHAC's and includes engagement of area and local-level inspection staff in the food safety investigation, food sampling and recall activities, and laboratory costs associated with food testing. This present analysis likely underestimates these costs. Likewise the costs for Health Canada were also a conservative estimate, as these were not calculated until after the conclusion of the outbreak and are based in part on best estimates available at the time. The costs associated with cases were estimated based on the case data provided by health authorities within each province, as well as our understanding of typical listeriosis cases. This did not include information on comorbidities that often accompany listeriosis, which may complicate diagnosis and treatment resulting in higher healthcare costs. In addition, some patients may have been required to travel to the hospital by ambulance, and these costs were not included. Thus, the estimated case costs likely represent an underestimate of the true burden. Furthermore, costs to industries and businesses other than the IMP were not considered and the estimated costs for the IMP were based on limited available information. With these limitations in mind, this study clearly highlights the large burden that foodborne disease and foodborne disease outbreaks represent to Canada, underscoring the importance of strong food safety policies and practices.

To our knowledge, no other listeriosis outbreak has been subject to a published cost analysis. Cost estimates for listeriosis based on total cases (i.e., sporadic and outbreak) have been completed for the United States (Medeiros et al., 2001; Batz et al., 2004, 2011; Hoffmann et al., 2012; Scharff, 2012), the Netherlands (Mangen et al., 2015), New Zealand (Scott et al., 2000; Cressey and Lake, 2008), and Australia (Abelson et al., 2006). In comparison with these international studies, the cost per case for the 2008 outbreak (including deaths, but excluding costs associated with federal outbreak response and the IMP) of approximately $\$ 2.8$ million (CAD 2008) is closest to, though higher than, the recent U.S. estimates ranging from $\$ 0.8$ to $\$ 1.7$ million (converted to CAD 2008) (Batz et al., 2004, 2011; Hoffmann et al., 2012; Scharff, 2012). Other international cost per case estimates for listeriosis are lower than the U.S. estimates and the Canadian estimate presented here, ranging from $\$ 11,000$ (CAD 2008) in New Zealand (Cressey and Lake, 2008) to $\$ 689,000$ (CAD 2008) in Australia (Abelson et al., 2006). This is likely a reflection of the large proportion of cases that died (42\%) in this outbreak, which involved particularly vulnerable populations in institutional settings and the high value of the cost per death recommended for use in Canadian policy analysis, which is one of the highest VSL values in the international literature (Chestnut and De Civita, 2009). Application of a lower VSL value for estimating the cost of death would result in a lower total cost per death and total cost per case estimate; the value used, however, is standard for cost analyses as per the Treasury Board of Canada Guidelines (http://www.tbs-sct.gc.ca/rtrapparfa/analys/analys-eng.pdf). Additional exploration of estimating societal costs associated with deaths is warranted.

In comparing case costs by component, the VSL value for premature deaths contributes the largest proportion to total costs, followed by costs associated with daily hospital fees. This is similar to the findings from studies conducted in the United States (Hoffmann et al., 2012) and Australia (Abelson et al., 2006). The sizeable costs associated with deaths relate to the economic value placed on a lost life. Given the ethical implications of placing a dollar value on lost life, another approach to estimating the burden associated with mortality is to use DALYs. In this study, we have estimated 2 DALYs lost per case, the sum of Years of Life Lost due to premature death and Years of Life Lived with Disability due to pain and suffering. This is similar to estimates from the United States, which measure the loss of healthy life due to listeriosis in Quality Adjusted Life Years (QALYs) lost, placing these values at approximately 3 (Hoffmann et al., 2012) and 3.5 (Batz et al., 2004).

Any costing exercise of this magnitude has its limitations. For the results here, many of the fees related to case cost inputs were based on the available information for the province of Ontario. Though cases occurred in other provinces, the majority $(72 \%)$ were located in Ontario. Other provincial cost information was not available, and it was assumed that any variability in fee structures that might occur between provinces would be captured in the uncertainty ranges of the final estimates. Future work would benefit from having individual provincial cost components. 
Assumptions were made for the most likely diagnostic and treatment procedures that would be applied to each case, as this level of information was not available for the specific outbreak cases. This information was reviewed by a listeriosis and clinical infectious disease specialist to verify that it was reasonable and appropriate given the level of case information available, as well as the typical presentation of listeriosis cases. However, comorbidities are often present in listeriosis cases and these might complicate the diagnosis and treatment of the disease. These costs were not explicitly incorporated into these estimates, representing an additional source of potential underestimation of the true cost of the outbreak.

Additionally, it is possible that cases traveled by ambulance to the hospital; these costs were not taken into account in these estimates. Also, costs associated with lost productivity or leisure time for patient caregivers were not incorporated, as this information was not available. The costs of pain and suffering in terms of financial loss were not incorporated; however, this is captured using the DALY estimates of Years of Life Lived with Disabilities.

Long-term consequences (i.e., chronic sequelae) that can result from listeriosis have been reported to include neurological and psychological conditions (Muller and Millard, 1976; Kemmeren et al., 2006; Batz et al., 2013; de Noordhout et al., 2014). Possible costs associated with these long-term consequences were not included in the final estimates, as there was not sufficient case information to support these estimates. We did explore possible sequelae that may have affected the recovered meningitis cases. However, given the low proportion of meningitis cases that typically develop sequelae and the low number of recovered meningitis cases, our estimates indicated that less than one case was likely to develop any type of chronic sequelae. Chronic sequelae remain a possible source of underestimation, and future cost of foodborne illness studies should explore how they can be incorporated.

There is discussion in the literature as to the best method for measuring lost productivity in cost of illness studies (Koopmanschap et al., 1995; Tranmer et al., 2005). In this study, the use of a modified friction cost method (described in detail in Supplementary Data S1) likely results in a smaller estimate than one resulting from a human capital valuation, which values the worker's lost productivity from the date of death until the expected age of retirement (Tranmer et al., 2005). Conversely, the traditional friction cost method values a worker's lost productivity at $80 \%$ of their expected wage (Koopmanschap et al., 1995), and so these estimates are likely to be slightly less than the estimate presented here.

The 2008 listeriosis outbreak represented a large public health and economic burden to Canadians. It emphasizes considerable consequences at both the individual and population levels associated with foodborne disease and foodborne disease outbreaks in particular. Future economic estimates of foodborne outbreaks would contribute to our understanding of the burden of foodborne disease in Canada and support the need for ongoing prevention and control activities. Prospective collection of cost data during outbreak management would assist in developing these types of estimates in the future. Canada's food safety system remains one of the best in the world. Continued efforts throughout the entire food continuum, from food production to the consumer, are needed to reduce the burden associated with foodborne disease.

\section{Acknowledgments}

The authors wish to acknowledge all members of the Public Health Agency of Canada, Health Canada, and the Canadian Food Inspection Agency for providing input on the time and resources spent in responding to this outbreak. In particular, we acknowledge the work of Ms. Kathleen Gropp for administering the human resources survey to Public Health Agency of Canada staff and Ms. Regan Murray for reviewing the manuscript. The authors also wish to acknowledge public health partners at the local and provincial level in the affected provinces for the investigative data collection that informed estimates of the costs related to outbreak cases.

\section{Disclosure Statement}

No competing financial interests exist.

\section{References}

Abelson P, Forbes MP, Hall G. The Annual Cost of Foodborne Illness in Australia. Canberra, ACT, Australia: Commonwealth of Australia, 2006, pp. 1-97.

Batz M, Hoffmann S, Krupnick A, Morris G, Sherman D, Taylor M, Tick J. Identifying the most significant microbiological foodborne hazards to public health: A new risk ranking model. Resources for the Future; 2004; Food Safety Research Consortium Discussion paper 1. Available at: http:// www.rff.org/files/sharepoint/WorkImages/Download/FRSCDP-01.pdf, accessed October 17, 2015.

Batz MB, Henke E, Kowalcyk B. Long-term consequences of foodborne infections. Infect Dis Clin North Am 2013;27: 599-616.

Batz MB, Hoffmann S, Morris JG Jr. Ranking the Risks: The 10 Pathogen-Food Combinations with the Greatest Burden on Public Health. Emerging Pathogens Institute, University of Florida, 2011.

Buzby JC, Roberts T, Lin CTJ, MacDonald JM. Bacterial Foodborne Disease: Medical Costs and Productivity Losses. Washington, DC: United States Department of Agriculture, Economic Research Service, 1996, p. 741.

Chestnut LG, De Civita P. Economic Valuation of Mortality Risk Reduction: Review and Recommendations for Policy and Regulatory Analysis. Ottawa, Ontario, Canada: Government of Canada Policy Research Initiative, 2009.

Cressey P, Lake R. Risk Ranking: Estimates of the Cost of Foodborne Disease for New Zealand. Christchurch, New Zealand: Institute of Environmental Science \& Research Limited, Client Report, 2008.

Currie A, Farber JM, Nadon C, Sharma D, Whitfield Y, Gaulin C, Galanis E, Bekal S, Flint J, Tschetter L, Pagotto F, Lee B, Jamieson F, Badiani T, MacDonald D, and the National Outbreak Investigation Team. Multi-province listeriosis outbreak linked to contaminated deli meat consumed primarily in institutional settings, Canada, 2008. Foodborne Pathog Dis 2015;12:645-652.

de Noordhout CM, Devleesschauwer B, Angulo FJ, Verbeke G, Haagsma J, Kirk M, Havelaar A, Speybroeck N. The global burden of listeriosis: A systematic review and meta-analysis. Lancet Infect Dis 2014;14:1073-1082.

Government of Canada. Canada's Food-borne Illness Outbreak Response Protocol (FIORP) 2010: To Guide a MultiJurisdictional Response. Government of Canada, 2010. 
Haagsma JA, van der Zanden BP, Tariq L, van Pelt W, van Duynhoven YTPH, Havelaar AH. Disease burden and costs of selected foodborne pathogens in The Netherlands, 2006. Rijksinstituut voor Volksgezondheid en Milieu (RIVM) Report. BA Bilthoven, The Netherlands: Ministry of Public Health, 2009.

Hoffmann S, Batz MB, Morris JG Jr. Annual cost of illness and quality-adjusted life year losses in the United States due to 14 foodborne pathogens. J Food Prot 2012;75:1292-1302.

Kemmeren JM, Mangen MJ, van Duynhoven YTHP, Havelaar AH. Priority setting of foodborne pathogens: Disease burden and costs of selected pathogens. Rijksinstituut voor Volksgezondheid en Milieu (RIVM)-Report. BA Bilthoven, The Netherlands: Ministry of Public Health, 2006.

Koopmanschap MA, Rutten FFH, van Ineveld BM, Van Roijen L. The friction cost method for measuring indirect costs of disease. J Health Econ 1995;14:171-189.

Mangen MJ, Bouwknegt M, Friesema IH, Haagsma JA, Kortbeek LM, Tariq L, Wilson M, van Pelt W, Havelaar AH. Cost-of-illness and disease burden of food-related pathogens in the Netherlands, 2011. Int J Food Microbiol 2015;196C: 84-93.

Medeiros LC, Hillers VN, Kendall PA, Mason A. Food safety education: What should we be teaching to consumers? J Nutr Educ Behav 2001;33:108-113.

Murray CJ. Quantifying the burden of disease: The technical basis for disability-adjusted life years. Bull World Health Organ 1994;72:429.

Rice DP. Cost of illness studies: What is good about them? Inj Prev 2000;6:177-179.

Roberts JA. Economic aspects of food-borne outbreaks and their control. Br Med Bull 2000;56:133-141.

Roberts JA, Sockett PN, Gill ON. Economic impact of a nationwide outbreak of salmonellosis: Cost-benefit of early intervention. BMJ 1989;298:1227-1230.

Roberts JA, Upton PA, Azene G. Escherichia coli O157:H7: Economic assessment of an outbreak. J Publ Health Med 2000;22:99-107.

Ryser ET, Marth EH. "New" food-borne pathogens of public health significance. J Am Diet Assoc 1989;89:948-954.
Scharff RL. Health-related costs from foodborne illnesses in the United States. The Produce Safety Project at Georgetown University, 2010, pp. 1-28.

Scharff RL. Economic burden from health losses due to foodborne illness in the United States. J Food Prot 2012;75:123-131.

Schlech WF, Acheson D. Foodborne listeriosis. Clin Infect Dis 2000;31:770-775.

Scott WG, Scott HM, Lake RJ, Baker MG. Economic cost to New Zealand of foodborne infectious disease. N Z Med J 2000;113:281-284.

Thomas MK, Murray R, Flockhart L, Pintar K, Pollari F, Fazil A, Nesbitt A, Marshall B. Estimates of the burden of foodborne illness in Canada for 30 specified pathogens and unspecified agents, circa 2006. Foodborne Pathog Dis 2013;10:639-648.

Todd ECD. Costs of acute bacterial foodborne disease in $\mathrm{Ca}$ nada and the United States. Int J Food Microbiol 1989a; 9:313-326.

Todd ECD. Preliminary estimates of costs of foodborne disease in Canada and costs to reduce salmonellosis. J Food Prot $1989 b ; 52: 586-594$.

Todd ECD. Economic loss from foodborne disease and nonillness related recalls because of mishandling by food processors. J Food Prot 1985;48:621-633.

Tranmer JE, Guerriere DN, Ungar WJ, Coyte PC. Valuing patient and caregiver time. Pharmacoeconomics 2005;23:449-459.

[WHO/FAO] World Health Organization and Food and Agriculture Organization of the United Nations. Risk assessment of Listeria monocytogenes in ready-to-eat foods: Microbiological Risk Assessment Series 5. Geneva and Rome: WHO/FAO, 2004.

\author{
Address correspondence to: \\ M. Kate Thomas, $M S c$, PhD \\ Public Health Agency of Canada \\ 120-255 Woodlawn Road West \\ Guelph, Ontario, N1H 8J1, Canada \\ E-mail: Kate.Thomas@phac-aspc.gc.ca
}

